A Universe of Dwarf Galaxies

Editors : will be set by the publisher

EAS Publications Series, Vol. ?, 2018

\title{
SATELLITES IN THE LOCAL GROUP AND OTHER NEARBY GROUPS
}

\author{
Eva K. Grebel ${ }^{1}$
}

\begin{abstract}
In recent years the census of known satellites in our own Local Group and in nearby galaxy groups has increased substantially due to sensitive wide-area surveys. In the Local Group these surveys have more than doubled its known galaxy content and extended the galaxy luminosity function to very faint total magnitudes. Deep ground-based imaging and spectroscopic observations as well as high-resolution imaging with the Hubble Space Telescope have revolutionized our understanding of the chemical evolution and star formation histories of the satellites. We often find long-lasting star formation episodes with low star formation efficiencies. There is evidence for localized, stochastic enrichment, and recent searches are now beginning to uncover even extremely metal-deficient stars. In many satellites evidence for two or more distinct stellar subpopulations is found. Differing fractions of old populations have been detected in all satellites studied in sufficient detail so far. Kinematic measurements support a picture in which satellites are dark-matter dominated, although recent results indicate that the proposed common mass scale for dwarf spheroidal galaxies may not hold for very low-mass satellites. When considering satellite ensembles, we find global morphology-distance and gas-content-distance relations in all groups studied thus far, but individual star formation histories also strongly depend on a given satellite's intrinsic properties.
\end{abstract}

\section{Introduction}

As noted by Karachentsev (2005), $85 \%$ of the galaxies in the Universe are located outside of rich galaxy clusters. About half of these galaxies are found in groups, and this fraction increases when also counting galaxies in unbound "clouds" and filaments. Such group environments are thus typical for the regions in which the

\footnotetext{
${ }^{1}$ Astronomisches Rechen-Institut, Zentrum für Astronomie der Universität Heidelberg, Mönchhofstr. 12-14, 69120 Heidelberg, Germany
} 
A Universe of Dwarf Galaxies

majority of galaxies currently live. They are also excellent laboratories to study galaxy evolution throughout the wide range of commonly found environments.

In this contribution I will focus on nearby groups of galaxies within about 5 $\mathrm{Mpc}$, which are close enough to permit us to resolve individual stars well below the tip of the red giant branch with only a modest investment of observing time with high-resolution cameras such as the ones aboard the Hubble Space Telescope (HST). These galaxies in the "Local Volume" have been targeted by several major observing campaigns. These include our 200-orbit HST snapshot program (Grebel 2000a) and the "ACS Nearby Galaxy Survey Treasury" (ANGST, Dalcanton et al. 2009) for resolved stellar populations, as well as ground-based broad-band and narrow-band imaging campaigns for integrated properties (e.g., Bremnes et al. 2000, Jerjen et al. 2000, Karachentsev et al. 2000, Barazza et al. 2001; Makarova et al. 2002a, 2005, Chiboucas et al. 2009, Côté et al. 2009, Fingerhut et al. 2010), ground-based studies of $\mathrm{H}$ I content and kinematics (e.g., Banks et al. 1999, Côté et al. 1997, 2000, Bouchard et al. 2005), and of (nebular) abundances (e.g., Richer et al. 1998, Skillman et al. 2003, Lee et al. 2003, 2007. Kniazev et al. 2005, 2008, Croxall et al. 2009). While these programs provide an increasingly elaborate picture of dwarf galaxy properties and evolution in nearby groups, the most detailed information is available for dwarf galaxies in the Local Group, particularly Milky Way companions, where we can even resolve stars below the oldest main-sequence turn-offs. Thus, special emphasis will be placed on the Local Group in this review.

Our focus on the Local Group and other nearby groups within $5 \mathrm{Mpc}$ implies that certain types of groups - in particular rich, fossil, or compact groups (see, e.g., Lee et al. 2004) - are excluded because we lack nearby examples. Nonetheless, we have a range of different group environments nearby, including strongly interacting and more quiescent groups, poor groups, and very loose groups or "clouds" (see also Tully 1987, Grebel 2007). The most commonly occurring dwarf galaxies in these nearby groups are dwarf elliptical (dE) and dwarf spheroidal (dSph) galaxies among the early-type dwarfs and dwarf irregular (dIrr) galaxies among the latetype dwarfs. Ultra-compact (e.g., Evstigneeva et al. 2007, Rejkuba et al. 2007) and compact dwarfs (e.g., Ziegler \& Bender 1998) and blue compact dwarfs (Gil de Paz et al. 2003, Kniazev et al. 2003, Richer et al. 2001) are rare. DEs and dSphs are usually satellites of the dominant, massive galaxies in groups, while dIrrs tend to be found at larger distances and may be bound to the group as a whole rather than to an individual high-mass galaxy. For a review of the properties of different types of dwarf galaxies, see Grebel (2001). - The interacting M81 group may even contain genuine tidal dwarf galaxies (Makarova et al. 2002b; Croxall et al. 2009, see Bournaud 2010 for a general review).

\section{Morphological Segregation and Environment}

Nearby galaxy groups within $5 \mathrm{Mpc}$ range from evolved groups like the Centaurus A group (e.g., Karachentsev et al. 2002a, 2007, Côté et al. 2009), the IC 342/Maffei group (e.g., Buta \& McCall[1999, Karachentsev et al. 2003a, Finger- 
hut et al. 2007) and the interacting M81 group (e.g., Yun et al. 1994 Karachentsev et al. 2002b; Chynoweth et al. 2008, Chiboucas et al. 2009) to little evolved, extended filaments or "clouds" such as the Sculptor and the Canes Venatici I group (e.g., Karachentsev et al. 2003b, 2003c). In addition, several sparse, possibly bound "dwarf groups" have been identified in the Local Volume (e.g., van den Bergh 1999a, Tully et al. 2006), whose most massive galaxy is usually an irregular.

The clouds or filaments contain primarily gas-rich, late-type galaxies that do not yet seem to have experienced many interactions or significant stripping of their gaseous material. The more evolved prominent galaxy groups typically contain two or more massive galaxies, each of which is surrounded by an entourage of lowermass satellites. Similar as in galaxy clusters (e.g., Oemler 1974, Dressler 1980, Lisker et al. 2007), where the fraction of early-type galaxies increases with galaxy density, in these groups as well as in the Local Group a clear morphology-density relation is observed (e.g., Einasto et al. 1974, van den Bergh 1999b, Grebel 1999, 2000b, 2005, and the above references). In particular, a pronounced increase in the number of early-type dwarfs is found within $\sim 300 \mathrm{kpc}$ around the massive galaxies in the more evolved groups. Environmental effects such as ram pressure, tidal forces, and galaxy harassment are believed to be responsible for the morphological segregation among dwarf galaxies (e.g., van den Bergh 1994, Grebel et al. 2003, Kravtsov et al. 2004; Mayer et al. 2006, D'Onghia et al. 2009; Grcevich \& Putman 2009). Also in more distant groups there is clear evidence both for morphological and for luminosity segregation (e.g., Girardi et al. 2003).

The morphology-density relation can be expressed as a morphology-radius relation or an H i mass-radius relation (e.g., Einasto et al. 1974; Grebel et al. 2003, Fig. 3). The upper limits of the H I masses for dwarf galaxies within $\sim 270 \mathrm{kpc}$ from the Milky Way or M31 are typically less than $10^{4} \mathrm{M}_{\odot}$ (Grcevich \& Putman 2009), and only the more massive dwarfs manage to retain some of their neutral gas at these close distances. Thus far there is no evidence for significant amounts of ionized gas in low-mass dSphs (e.g., Gallagher et al. 2003). These findings contrast with the low-density, extended Sculptor group filament, where many of the early-type dwarfs were found to have H I masses of several $10^{5} \mathrm{M}_{\odot}$ (Bouchard et al. 2005), seemingly having been able to retain gas from normal stellar outflows in the absence of external stripping. On the other hand, ram pressure stripping appears to be at work in the higher-density Cen A group (Bouchard et al. 2007).

\section{Faint Satellite Luminosities, Masses, and the Substructure Problem}

One of the long-standing unsolved questions in cosmology is the substructure problem - the problem that cosmological simulations predict up to two orders of magnitude more small dark matter halos than the number of actually observed satellite galaxies (e.g., Klypin et al. 1999, Moore et al. 1999, Kravtsov 2010). Many scenarios have been suggested in order to resolve this apparent discrepancy. Among others, these scenarios include the existence of dark matter halos without baryons due to early photoevaporation, possibly during reionization or because of various feedback effects (e.g., Bullock et al. 2000, Madau et al. 2008, Wadepuhl \& 
Springel 2011), a substantial underestimate of the observed circular velocities of the satellites (e.g., Stoehr et al. 2002, Peñarrubia et al. 2008), or an observational bias in detecting faint satellites (e.g., Tollerud et al. 2008, Willman 2010).

Indeed the galaxy census of the Local Group and of nearby groups is significantly incomplete toward the faint end of the galaxy luminosity function. In recent years, a considerable number of new satellites was discovered both in the Local Group (e.g., Zucker et al. 2004a, 2006a, 2006b, 2007, Belokurov et al. 2006, 2007, 2010, Willman et al. 2005, Martin et al. 2006, 2009, Chapman et al. 2007, Walsh et al. 2007, Majewski et al. 2007, McConnachie et al. 2008) and in nearby groups (e.g., Côté et al. 1997, Karachentseva \& Karachentsev 1998, Banks et al. 1999, Chiboucas et al. 2009). More sensitive surveys and wider sky coverage should reveal even more faint dwarfs (see Tollerud et al. 2008), alleviating the "missing satellite problem". On the other hand, the higher resolution of modern cosmological simulations tends to predict even more small dark matter halos, worsening the discrepancy again (Madau et al. 2008, Bovill \& Ricotti 2009).

Most of the newly discovered dwarfs are dim dSphs. These include the "ultrafaint" dSphs (mostly Milky Way satellites), the faintest galaxies known with luminosities down to $\sim 10^{3} \mathrm{~L}_{\odot}$. The new Local Group dwarfs are mainly dominated by old populations and are typically quite metal-poor (e.g., Whiting et al. 1999; Grebel \& Guhathakurta 1999, Harbeck et al. 2004, 2005, Simon \& Geha 2007. Kirby et al. 2008, Koch et al. 2008a, Kalirai et al. 2009, Frebel et al. 2010a, Adén et al. 2011, and references above). Such dwarf galaxies that formed most of their stars at $z \sim 8$ could represent "fossils" of the era of reionization (e.g., Gnedin \& Kravtsov 2006, Bovill \& Ricotti 2009). While the current detections in other groups or even around M31 do not yet reach as faint a luminosity as in the new Milky Way companions, the faint-end slope of the cumulative luminosity functions in nearby groups appears to be rather similar, typically of the order of $-1.3 \pm 0.1$, apparently independent of environment (Chiboucas et al. 2009).

Interestingly, the radial velocity dispersion profiles of $\mathrm{dSphs}$ are fairly flat as a function of radius and reveal high velocity dispersions even at large radii, which is usually interpreted as an indication of a high dark matter content (e.g., Wilkinson et al. 2004; Koch et al. 2007a, 2007b; Gilmore et al. 2007a; Walker et al. 2007, Ural et al. 2010). In fact, it has been suggested that all dSph galaxies may share a common halo mass (e.g., Gilmore et al. 2007a, Strigari et al. 2008, Walker et al. 2009, Wolf et al. 2010), and that perhaps even the central surface density of dark matter halos is approximately constant and independent of galaxy luminosity (e.g., Donato et al. 2009). However, there are also indications of deviations from a uniform enclosed mass out to a constant radius: Some measurements suggest that there is a decline in halo mass with decreasing luminosity after all. Careful removal of interloper stars in sparse stellar systems leads to lower velocity dispersions and masses (Adén et al. 2009a, 2009b). Also, there may be intrinsic differences between M31 and Milky Way dSph satellites, and some some of the ultra-faint dSphs may be experiencing tidal disruption (e.g., Kalirai et al. 2010, Collins et al. 2010, Simon \& Geha 2007, but see Peñarrubia et al. 2008 for a different view).

Generally, caution is advisable when interpreting morphological features as 
signatures of tidal disruption (Muñoz et al. 2008). Thus far no evidence of Galactic dSphs being unbound tidal remnants with a large line-of-sight depth has been found (Klessen et al. 2003). In any case, the existing stellar radial velocity profiles probably still only trace the dwarfs' inner regions; at larger distances a decline is expected (e.g., Prada et al. 2003). Deeper surveys do indeed occasionally reveal that even the luminous extent of dwarf galaxies is larger than previously assumed (e.g., Odenkirchen et al. 2001; Komiyama et al. 2003, Kniazev et al. 2009).

Velocity dispersion profiles appear to indicate a preference for cored profiles in dSphs (e.g., Gilmore et al. 2007b, Battaglia et al. 2008), although cuspy dark matter profiles are not excluded. Observations also seem to support constant dark matter densities in the central regions of galaxies (e.g., Donato et al. 2009), whereas cosmological simulations predict steeply rising profiles. This "core/cusp" problem is another unsolved problem in modern cosmology (see de Blok 2010 for a review). A promising solution of this long-standing problem may be that initially cuspy dark matter profiles become cored simply as a consequence of star formation and feedback, resolving the contradiction (e.g., Pasetto et al. 2010).

\section{Star Formation Histories and Chemical Evolution}

\subsection{Global star formation histories}

Global star-formation rates of galaxies in the present-day Universe tend to increase with decreasing stellar mass, but show increasing scatter when entering the lowluminosity dwarf regime (Bothwell et al. 2009). Many late-type dwarf galaxies have a sufficiently high $\mathrm{H}$ I content to continue to form stars for another Hubble time, but still show only very low star-formation rates, which may be due to very low gas densities that increase the H I consumption time scales (see also Hunter 1997). Low-efficiency star formation may, however, evade detection as it would be difficult to trace with commonly used techniques such as $\mathrm{H} \alpha$ emissivity (Bothwell et al. 2009). Apart from the range of present-day star formation properties in latetype dwarfs, the scatter in star-formation rates at the low-mass end of the baryonic mass distribution is further increased by the presence of quiescent, gas-deficient early-type dwarfs.

In the Local Group no two dwarf galaxies share the same star formation history, not even within the same morphological type (Grebel 1997, 1999, see also Orban et al. 2008). Despite their differences, dwarfs share many common evolutionary trends and follow similar scaling relations (e.g., Sharina et al. 2008). Apart from morphological segregation, the star formation rates and star formation histories of dwarfs in the Local Group and in nearby groups do not show a clear correlation with distance from the closest massive primary (e.g., Weisz et al. 2011, Côté et al. 2009, Crnojević et al. 2010, Lianou et al. 2010). This suggests that intrinsic properties rather than environment may govern the evolution of dwarf galaxies (see also Revaz et al. 2009). However, the orbits of these dwarfs are unknown. Models considering realistic orbits (for Milky Way companions) support the triggering of star formation activity in dwarfs through encounters (e.g., Pasetto et al. 2011). 


\subsection{Old stellar populations}

All Local Group dwarfs studied in detail thus far show evidence for very old populations (e.g., Grebel \& Gallagher 2004) as demonstrated by the presence of old, age-dateable main-sequence turn-offs in field populations or globular clusters, horizontal branches, and/or RR Lyrae stars. The fraction of old stars varies considerably from galaxy to galaxy. An example of a dIrr galaxy with a very modest old population is the Small Magellanic Cloud (SMC): Its only globular cluster is about two billion years younger than typical old globulars in the Large Magellanic Cloud, Fornax, Sagittarius, and the Milky Way (e.g., Glatt et al. 2008a). RR Lyrae stars were detected in the SMC in much smaller numbers than in the neighboring Large Magellanic Cloud (Soszyński et al. 2010). In a few other Local Group dIrrs the existence of an ancient population was questioned until finally RR Lyrae stars were detected (e.g., Dolphin et al. 2002). In contrast, early-type dwarfs typically contain rather prominent old populations.

From their analysis of 60 Local Volume dwarfs with color-magnitude diagrams reaching the upper red giant branch, Weisz et al. (2011) infer that, on average, dwarf galaxies formed more than $50 \%$ of their stars by $z \sim 2$ (see also Crnojević et al. 2011). Unambiguous evidence for ancient populations beyond the Local Group, however, so far only exists for two dwarfs in the Sculptor group, where blue horizontal branches and RR Lyrae stars were detected (Da Costa et al. 2010).

\subsection{Abundance spreads and inhomogeneous enrichment}

Dwarf galaxies typically reveal indications of extended episodes of star formation leading to considerable abundance spreads (of one dex in $[\mathrm{Fe} / \mathrm{H}]$ or more) even in purely old populations (see, e.g., Shetrone et al. 2001, Grebel et al. 2003, Cohen \& Huang 2010). While we lack stellar spectroscopy for galaxies beyond the Local Group, the broad red giant branches of dwarfs in nearby groups suggest the existence of considerable metallicity spreads there as well (e.g., Sharina et al. 2008, Crnojević et al. 2010, Lianou et al. 2010) and can be translated into photometric metallicity distribution functions. For Local Group satellites a growing number of spectroscopic metallicity distribution functions is available (e.g., Helmi et al. 2006, Koch et al. 2006, 2007b, 2007c; Kirby et al. 2008), confirming large spreads.

In some nearby galaxies evidence for metallicity spreads has been found even within coeval populations (e.g., Venn et al. 2003, Kniazev et al. 2005, Koch et $a l$. 2007c). This is particularly pronounced in the SMC, where metallicity spreads seem to exist at any given age (Glatt et al. 2008b). There is also evidence for inhomogeneous enrichment among stars of the same metallicity. Individual element abundance ratios may permit one to even trace individual supernova events (e.g., Sadakane et al. 2004, Koch et al. 2008a, 2008b; Adén et al. 2011).

Abundance inhomogeneities are usually attributed to slow, stochastic, localized star formation with low star formation efficiency (e.g., Marcolini et al. 2008). These models also predict the usually observed asymmetric metallicity distribution functions with an extend low-metallicity tail, a slow rise toward higher metallicities 
and subsequently a rapid fall-off. Alternatively, there are successful models in which star formation rates and galactic wind efficiencies are chosen to reproduce the observed metallicity distributions (e.g., Lanfranchi \& Matteucci 2007, 2010).

Overall, the dwarf galaxies in the Local Group as well as those in nearby groups follow similar metallicity-luminosity relations, and there appears to be a general trend for dSphs being "too metal-rich" for their luminosity as compared to latetype dwarfs (e.g., Richer et al. 1998, Grebel et al. 2003, Sharina et al. 2008). This interesting trend may indicate that early-type dwarfs experienced more efficient and rapid enrichment early on, while the chemical evolution of late-type dwarfs, which essentially experienced continuous star formation (Cignoni \& Tosi 2010), was slower and less efficient. As discussed in Grebel et al. (2003), this difference makes it more difficult to turn dIrrs into dSphs. But low-mass transition-type galaxies may be plausible progenitors of dSphs in terms of their stellar population properties, metallicities, and star-formation histories.

\subsection{Stellar population gradients}

Many, but not all, dwarf galaxies exhibit population gradients. When gradients are present, the more metal-rich and/or younger populations are usually found to be more centrally concentrated, while older and/or more metal-poor populations have a more extended, smoother distribution and may show distinct kinematics with a higher velocity dispersion (e.g., Grebel 1997, Hurley-Keller et al. 1999, Zaritsky et al. 2000, Harbeck et al. 2001, Tolstoy et al. 2004, Koch et al. 2006, Weisz et al. 2011, Leaman et al. 2009, Crnojević et al. 2010, Lianou et al. 2010, Battaglia et al. 2011). As these studies show, population gradients are found both in early- and late-type dwarfs in the Local Group and nearby groups and are well-reproduced by models (e.g., Marcolini et al. 2008).

\subsection{Detailed element abundance ratios and satellite accretion}

Satellite galaxies may ultimately get accreted by the massive galaxies that they orbit. There is mounting evidence of such events in the Local Group, both in the form of disruption and accretion streams (e.g., Ibata et al. 1994, Newberg et al. 2003, Yanny et al. 2003, McConnachie et al. 2009, Williams et al. 2011) and as substructure in the Galactic or M31 halo (e.g., Bell et al. 2008 Zucker et al. 2004b). Interactions are clearly also playing a role in the M81 and the Cen A groups. However, it remains unclear to what extent observable satellites may have contributed to the build-up of massive galaxies, particularly their halos (see, e.g., Unavane et al. 1996, Bullock et al. 2001; Robertson et al. 2005; Font et al. 2008).

DSphs tend to reach solar $[\alpha / \mathrm{Fe}]$ ratios already at much lower metallicities than Galactic halo stars (the details vary from dwarf to dwarf depending on, e.g., the initial gas mass; Pasetto et al. 2010$)$. The lower $[\alpha / \mathrm{Fe}]$ ratio at a given $[\mathrm{Fe} / \mathrm{H}]$ has been attributed to several possible factors including low star formation rates (and

hence little $\alpha$ enrichment from massive supernovae of type II), or to efficient loss of metals and supernova ejecta due to the shallow potential wells of the dwarfs, 
or/and to a larger contribution of Fe from supernovae of type Ia (Shetrone et al. 2001). Ultimately, the $[\alpha / \mathrm{Fe}]$ ratio may be viewed as a tracer of the accretion time. At low metallicities (or early accretion) dSphs and Galactic halo stars are in very good agreement (see also below).

Nonetheless, the difference in $[\alpha / \mathrm{Fe}]$ ratios at a given $[\mathrm{Fe} / \mathrm{H}]$ in $\mathrm{dSphs}$ and in the Galactic halo as well as the apparent lack of extremely metal-poor stars in dSphs were used to argue against a significant contribution of low-mass satellites to the build-up of the Galactic halo, suggesting a different chemical evolution history (e.g., Shetrone et al. 2001; Helmi et al. 2006, Koch et al. 2006, 2007c). This picture is now changing.

Many recent studies (e.g., Koch et al. 2008b, Kirby et al. 2008, Aoki et al. 2009, Cohen \& Huang 2009, 2010, Frebel et al. 2010a, 2010b, Tafelmeyer et al. 2010) successfully detected very metal-deficient stars in classical and ultra-faint dSphs. Furthermore, these studies emphasize the similarity to very metal-poor stars in the Galactic halo, proposing that the chemical properties of low-mass dwarf satellites are well consistent with the stellar properties of especially the outer halo. Moreover, Nissen \& Schuster (2010) detected two kinematically distinct populations of halo stars in the solar neighborhood, one with high $[\alpha / \mathrm{Fe}]$ ratios and one with low $[\alpha / \mathrm{Fe}]$ ratios, which they attribute to objects formed in the Milky Way and to objects accreted from dwarf galaxies.

Forthcoming new photometric and spectroscopic surveys (including the Gaia satellite) should help to elucidate the contribution of satellites to more massive galaxies. Clearly, dwarf satellites play a number of important roles: They are interesting in their own right, revealing a wide range of properties that still provide a puzzle for a proper understanding of galaxy evolution, e.g., regarding the question of "nature" vs. "nurture". They provide valuable insights into stellar evolution at low metallicities. They may be key objects for understanding dark matter. They may play an important role as building blocks of more massive galaxies. And they are important test objects for cosmological theories, currently still challenging our ability to understand structure formation on small scales.

\section{References}

Adén, D., et al. 2009a, A\&A, 506, 1147

Adén, D., et al. 2009b, ApJ, 706, L150

Adén, D., et al. 2011, A\&A, 525, 153

Aoki, W., et al. 2009, A\&A, 502, 569

Banks, G.D., et al. 1999, ApJ, 524, 612

Barazza, F.D., Binggeli, B., Prugniel, P. 2001, A\&A, 373, 12

Battaglia, G., et al. 2011, MNRAS, 411, 1013

Battaglia, G., et al. 2008, ApJ, 681, L13

Bell, E.F., et al. 2008, ApJ, 680, 295

Belokurov, V., et al. 2006, ApJ, 647, L111

Belokurov, V., et al. 2007, ApJ, 654, 897 
Belokurov, V., et al. 2010, ApJ, 712, L103

Bothwell, M.S., Kennicutt, R.C., Lee, J.C. 2009, MNRAS, 400, 154

Bouchard, A., Jerjen, H., Da Costa, G., Ott, J. 2005, AJ, 130, 2058

Bouchard, A., Jerjen, H., Da Costa, G.S., Ott, J. 2007, AJ, 133, 261

Bournaud, F. 2010, AdAst, 735284

Bovill, M.S., Ricotti, M. 2009, ApJ, 693, 1859

Bremnes, T., Binggeli, B., Prugniel, P. 2000, A\&AS, 141, 211

Bullock, J.S., Kravtsov, A.V., Weinberg, D.H. 2000, ApJ, 539, 517

Bullock, J.S., Kravtsov, A.V., Weinberg, D.H. 2001, ApJ, 548, 33

Buta, R.J., McCall, M.L. 1999, ApJS, 124, 33

Chapman, S.C., et al. 2007, ApJ, 662, L79

Chiboucas, K., Karachentsev, I.D., Tully, R.B. 2009, AJ, 137, 3009

Chynoweth, K.M., et al. 2008, AJ, 135, 1983

Cignoni, M., Tosi, M. 2010, AdAst, 158568

Cohen, J.G., Huang, W. 2009, ApJ, 701, 1053

Cohen, J.G., Huang, W. 2010, ApJ, 719, 931

Collins, M.L.M., et al. 2010, MNRAS, 407, 2411

Côté, S., Freeman, K.C., Carignan, C., Quinn, P.J. 1997, AJ, 114, 1313

Côté, S., Carignan, C., Freeman, K.C. 2000, AJ, 120, 3027

Côté, S., Draginda, A., Skillman, E.D., Miller, B.W. 2009, AJ, 125, 610

Crnojević, D., Grebel, E.K., Koch, A. 2010, A\&A, 516, 85

Crnojević, D., Grebel, E.K., Cole, A.A. 2011, A\&A, in press (arXiv:1103.3707)

Croxall, K.V., et al. 2009, ApJ, 705, 723

Dalcanton, J.J., et al. 2009, ApJS, 183, 67

Da Costa, G.S., Rejkuba, M., Jerjen, H., Grebel, E.K. 2010, ApJ, 708, L121

de Blok, W.J.G., 2010, AdAst, 789293

Dolphin, A.E., et al. 2002, AJ, 123, 3154

Donato, F., et al. 2009, MNRAS, 397, 1169

D’Onghia, E., Besla, G., Cox, T.J., Hernquist, L. 2009, Nature, 460, 605

Dressler, A. 1980, ApJ, 236, 351

Evstigneeva, E.A., et al. 2007, MNRAS, 378, 1036

Einasto, J., Saar, E., Kaasik, A., Chernin, A.D. 1974, Nature, 252, 111

Fingerhut, R.L., Lee, H., McCall, M., Richer, M.G. 2007, ApJ, 655, 814

Fingerhut, R.L., et al. 2010, ApJ, 716, 792

Font, A.S., et al. 2008, 673, 215

Frebel, A., Simon, J.D., Geha, M., Willman, B. 2010a, ApJ, 708, 560

Frebel, A., Kirby, E.N., Simon, J.D. 2010b, Nature, 464, 72

Gallagher, J.S., et al. 2003, ApJ, 588, 326

Gil de Paz, A., Madore, B.F., Pevunova, O. 2003, ApJS, 147, 29

Gilmore, G., et al. 2007a, ApJ, 663, 948

Gilmore, G., et al. 2007b, NuPhS, 173, 15

Girardi, M., Rigoni, E., Mardirossian, F., Rigoni, E. 2003, A\&A, 406, 403 
Glatt, K., et al. 2008a, AJ, 135, 1106

Glatt, K., et al. 2008b, AJ, 136, 1703

Gnedin, N.Y., Kravtsov, A.V. 2006, ApJ, 645, 1054

Grebel, E.K. 1997, Reviews in Modern Astronomy, 10, 29

Grebel, E.K. 1999, in The Stellar Content of the Local Group, IAU Symp. 192, P. Whitelock \& R. Cannon, eds., (San Francisco: ASP), 17

Grebel, E.K. 2000a, in Stars, Gas, and Dust in Galaxies: Exploring the Links, ASP Conf. Ser. 221, D. Alloin, K. Olsen, \& G. Galaz, eds., (San Francisco: ASP), 147

Grebel, E.K. 2000b, in Star Formation from the Small to the Large Scale, 33rd ESLAB Symp., ESA SP-445, F. Favata, A.A. Kaas, \& A. Wilson, eds. (Noordwijk: ESA), 87

Grebel, E.K. 2001, ApSSS, 277, 231

Grebel, E.K. 2005, in Stellar Astrophysics with the World's Largest Telescopes, AIP Conf. Proc. 752, J. Mikołaewska \& A. Olech, eds., (Melville: AIP), 161

Grebel, E.K. 2007, in "Groups of Galaxies in the nearby Universe, ESO Astrophysics Symposia, I. Saviane, V.D. Ivanov, \& J. Borissova, eds., (Berlin: Springer Verlag), 3

Grebel, E.K., Guhathakurta, P. 1999, ApJ, 511, L101

Grebel, E.K., Gallagher, J.S., Harbeck, D. 2003, AJ, 125, 1926

Grebel, E.K., Gallagher, J.S. 2004, ApJ, 610, L89

Grcevich, J., Putman, M.E. 2009, ApJ, 696, 385

Harbeck, D., et al. 2001, AJ, 122, 3092

Harbeck, D., et al. 2004, AJ, 127, 2711

Harbeck, D., et al. 2005, ApJ, 623, 159

Helmi, A., et al. 2006, ApJ, 651, L121

Hunter, D., 1997, PASP, 109, 937

Hurley-Keller, D., Mateo, M., Grebel,E.K. 1999, ApJ, 523, L25

Ibata, R.A., Gilmore, G., Irwin, M.J. 1994, Nature, 370, 194

Jerjen, H., Binggeli, B., Freeman, K.C. 2000, AJ, 119, 593

Kalirai, J.S., et al. 2009, ApJ, 705, 1043

Kalirai, J.S., et al. 2010, ApJ, 711, 671

Karachentsev, I.D., et al. 2000, A\&AS, 145, 415

Karachentsev, I.D., et al. 2002a, A\&A, 385, 21

Karachentsev, I.D., et al. 2002b, A\&A, 383, 125

Karachentsev, I.D., et al. 2003a, A\&A, 408, 111

Karachentsev, I.D., et al. 2003b, A\&A, 404, 93

Karachentsev, I.D., et al. 2003c, A\&A, 398, 467

Karachentsev, I.D. 2005, AJ, 129, 178

Karachentsev, I.D., et al. 1007, AJ, 133, 504

Karachentseva, V.E., Karachentsev, I.D. 1998, A\&AS, 127, 409

Kirby, E.N., et al. 2008, ApJ, 685, L43

Klessen, R.S., Grebel, E.K., Harbeck, D. 2003, ApJ, 589, 798

Klypin, A., Kravtsov, A.V., Valenzuela, O., Prada, F. 1999, ApJ, 522, 82

Kniazev, A.Y., et al. 2003, ApJ, 593, L73

Kniazev, A.Y., et al. 2005, AJ, 130, 1558 
Kniazev, A.Y., et al. 2008, MNRAS, 388, 1667

Kniazev, A.Y., et al. 2009, MNRAS, 400, 2054

Koch, A., et al. 2006, AJ, 131, 895

Koch, A., et al. 2007a, AJ, 134, 566

Koch, A., et al. 2007b, ApJ, 657, 241

Koch, A., et al. 2007c, AJ, 133, 270

Koch, A., et al. 2008a, ApJ, 688, L13

Koch, A., et al. 2008b, AJ, 135,1580

Komiyama, Y., et al. 2003, ApJ, 590, L17

Kravtsov, A. 2010, AdAst, 281913

Kravtsov, A.V., Gnedin, O.Y., Klypin, A.A. 2004, ApJ, 609, 482

Lanfranchi, G.A., Matteucci, F. 2007, A\&A, 927

Lanfranchi, G.A., Matteucci, F. 2010, A\&A, 512, 85

Leaman, R., et al. 2009, ApJ, 699, 1

Lee, B.C., et al. 2004, AJ, 127, 1811

Lee, H., Grebel, E.K., Hodge, P.W. 2003, A\&A, 401, 141

Lee, H., Zucker, D.B., Grebel, E.K. 2007, MNRAS, 376, 820

Lianou, S., Grebel, E.K., Koch, A. 2010, A\&A, 521, 43

Lisker, T., Grebel, E.K., Binggeli, B., Glatt, K. 2007, ApJ, 660, 1186

Madau, P., Diemand, J., Kuhlen, M. 2008, ApJ, 679, 1260

Majewski, S.R., et al. 2007, ApJ, 670, L9

Makarova, L.N., et al. 2002a, A\&A, 384, 72

Makarova, L.N., et al. 2002b, A\&A, 396, 473

Makarova, L.N., et al. 2005, A\&A, 433, 751

Marcolini, A., D'Ercole, A., Battaglia, G., Gibson, B.K. 2008, MNRAS, 386, 2173

Martin, N.F., et al. 2006, MNRAS, 371, 1983

Martin, N.F., et al. 2009, ApJ, 705, 758

Mayer, L., et al. 2006, MNRAS, 369, 1021

McConnachie, A.W., et al. 2008, ApJ, 688, 1009

McConnachie, A.W., et al. 2009, Nature, 461, 66

Moore, B., et al. 1999, ApJ, 524, L19

Muñoz, R.R., Majewski, S.R., Johnston, K.V. 2008, ApJ, 679, 346

Newberg, H.J., et al. 2003, ApJ, 596, L191

Nissen, P.E., Schuster, W.J. 2010, A\&A, 511, L10

Odenkirchen, M., et al. 2001, AJ, 122, 2538

Oemler, A. 1974, ApJ, 194, 1

Orban, C., et al. 2008, ApJ, 686, 1030

Pasetto, S., et al. 2010, A\&A, 514, 47

Pasetto, S., et al. 2011, A\&A, 525, 99

Peñarrubia, J., McConnachie, A.W., Navarro, J.F. 2008, ApJ, 672, 904

Prada, F., et al. 2003, ApJ, 598, 260

Rejkuba, M., Dubath, P., Minniti, D., Meylan, G. 2007, A\&A, 469, 147 
Revaz, Y., et al. 2009, A\&A, 501, 189

Richer, M.G., McCall, M.L., Stasińska, G. 1998, A\&A, 340, 67

Richer, M.G., et al. 2001, A\&A, 370, 34

Robertson, B., et al. 2005, ApJ, 632, 872

Sadakane, K., et al. PASJ, 56,1041

Sharina, M.E., et al. 2008, MNRAS, 384, 1544

Shetrone, M.D., Côté, P., Sargent, W.L.W. 2001, ApJ, 548, 592

Simon, J.D., Geha, M. 2007, ApJ, 670, 313

Skillman, E.D., Côté, S., Miller, B.W. 2003, AJ, 125, 610

Soszyński, I., et al. 2010, AcA, 60, 165

Stoehr, F., White, S.D.M., Tormen, G., Springel, V. 2002, MNRAS, 335, L84

Strigari, L.E., et al. Nature, 454, 1096

Tafelmeyer, M., et al. A\&A, 524, 58

Tollerud, E.J., Bullock, J.S., Strigari, L.E., Willman, B. 2008, ApJ, 688, 277

Tolstoy, E., et al. 2004, ApJ, 617, L119

Tully, R.B. 1987, ApJ, 321, 280

Tully, R.B., et al. 2006, AJ, 132, 729

Unavane, M., Wyse, R.F.G., Gilmore, G. 1996, MNRAS, 278, 727

Ural, U., et al. 2010, MNRAS, 402, 1357

van den Bergh, S. 1994, ApJ, 428, 617

van den Bergh, S. 1999a, ApJ, 517, L97

van den Bergh, S. 1999b, AJ, 117, 2211

Venn, K.A., et al. 2003, AJ, 126, 1326

Wadepuhl, M., Springel, V. 2011, MNRAS, 410, 1975

Walker, M.G., et al. 2007, ApJ, 667, L53

Walker, M.G., et al. 2009, ApJ, 704, 1274

Walsh, S.M., Jerjen, H., Willman, B. 2007, ApJ, 662, L83

Weisz, D.R., et al. 2008, ApJ, 689, 160

Weisz, D.R., et al. 2011, ApJ, submitted (arXiv:1101.1093)

Whiting, A.B., Hau, G.K.T., Irwin, M. 1999, AJ, 118, 2767

Williams, M.E.K., et al. 2011, ApJ, 728, 102

Wilkinson, M.I., et al. 2004, ApJ, 611, L21

Willman, B., et al. 2005, ApJ, 626, L85

Willman, B. 2010, AdAst, 285454

Wolf, J., et al. 2010, MNRAS, 406, 1220

Yanny, B., et al. 2003, ApJ, 588, 824

Yun, M.S., Ho, P.T.P., Lo, K.Y. 1994, Nature, 372, 530

Zaritsky, D., Harris, J., Grebel, E.K., Thompson, I.B. 2000, ApJ, 534, L53

Ziegler, B.L., Bender, R. 1998, A\&A, 330, 819

Zucker, D.B., et al. 2004a, ApJ, 612, L121

Zucker, D.B., et al. 2004b, ApJ, 612, L117

Zucker, D.B., et al. 2006a, ApJ, 643, L103

Zucker, D.B., et al. 2006b, ApJ, 650, L41

Zucker, D.B., et al. 2007, 659, L21 